\title{
Altered structural covariance and functional connectivity of the insula in patients with Crohn's disease
}

\author{
Shuming Zhang ${ }^{1,2}$, Fenrong Chen ${ }^{3}$, Jiayu Wu ${ }^{1,2}$, Chengxiang Liu ${ }^{1,2}$, Guang Yang ${ }^{1,2}$, Ruiqing Piao ${ }^{1,2}$, \\ Bowen Geng ${ }^{1,2}, \mathrm{Ke} \mathrm{Xu}^{1,2}$, Peng Liu ${ }^{1,2}$ \\ ${ }^{1}$ Life Science Research Center, School of Life Science and Technology, Xidian University, Xi'an, China; ${ }^{2}$ Engineering Research Center of Molecular \\ and Neuroimaging, Ministry of Education, Xi'an, China; ${ }^{3}$ Department of Gastroenterology, Second Affiliated Hospital of Xi'an Jiaotong University, \\ Xi'an, China \\ Contributions: (I) Conception and design: P Liu; (II) Administrative support: P Liu; (III) Provision of study materials or patients: F Chen; (IV) \\ Collection and assembly of data: S Zhang; (V) Data analysis and interpretation: S Zhang; (VI) Manuscript writing: All authors; (VII) Final approval \\ of manuscript: All authors.
}

Correspondence to: Peng Liu. Life Sciences Research Center, School of Life Science and Technology, Xidian University, Xi'an 710071, China. Email: liupengphd@gmail.com.

Background: Crohn's disease (CD) is a clinically chronic inflammatory bowel disease, which has been shown to be closely related to the brain-gut axis dysfunction. Although traditionally considered to be a limbic region, the insula has also been commonly identified as an abnormal brain region in previous CD-related studies.

Methods: Structural magnetic resonance imaging (MRI) and resting-state functional MRI images were acquired from $45 \mathrm{CD}$ patients in remission and 40 healthy controls (HCs). Three neuroimaging analysis methods including voxel-based morphometry (VBM), structural covariance, and functional connectivity (FC) were applied to investigate structural and functional alterations of the insulae between the CD patients and HCs. Pearson correlation was then used to examine the relationships between neuroimaging findings and clinical symptoms.

Results: Compared with the HCs, CD patients exhibited decreased gray matter volume (GMV) in the left dorsal anterior insula (dAI) and bilateral posterior insula (PI). Taking these three areas including the left dAI, right PI, and left PI as regions of interest (ROIs), differences were observed in the structural covariance and FC of the ROI with several regions between the two groups. After controlling for psychological factors, the differences of several regions involved in emotional processing in GMV in the left dAI, the FC of the dAI, and the right PI were not significant. The FC of the parahippocampus/hippocampus with dAI and PI were negatively correlated with the CD activity index (CDAI).

Conclusions: We suggest that the insula-centered structural and/or functional changes may be associated with abnormal visceral sensory processing and related emotional responses in CD patients.

Keywords: Crohn's disease (CD); insula; functional connectivity (FC); structural covariance; magnetic resonance imaging (MRI)

Submitted May 12, 2021. Accepted for publication Sep 01, 2021.

doi: 10.21037/qims-21-509

View this article at: https://dx.doi.org/10.21037/qims-21-509 


\section{Introduction}

Crohn's disease (CD) is a chronic inflammatory disease, which may manifest anywhere in the gastrointestinal tract from the mouth to the anus, and has shown a global increase in incidence (1). Symptoms often include abdominal pain, diarrhea, fever, and weight loss (1). These symptoms have a significant impact on patients' health-related quality of life, and CD patients have a slightly reduced life expectancy (2). As a lifelong disease, $\mathrm{CD}$ might result from a complex interplay between genetic susceptibility, environmental factors, and altered gut microbiota, leading to dysregulated innate and adaptive immune responses (1). Under physiological and pathological conditions, the brain and gut communicate via the autonomic nervous system (ANS) and circumventricular organs, which constitute the neuroanatomical basis of brain-gut interaction (3-5). Previous neuroimaging studies have indicated that the brain-gut axis plays a critical role in regulating gut sensation and bowel movement control, with dysfunction of the axis being associated with the changed perception of the nervous system, which might influence gastrointestinal symptoms $(3,6,7)$.

As functional and structural brain magnetic resonance imaging (MRI) technologies become increasingly popular, brain abnormalities are being more frequently discovered in CD-related neuroimaging studies. For example, compared with healthy controls (HCs), CD patients were reported to have changed gray matter volume (GMV) in various brain regions, including the insula, thalamus, and amygdala (8). The cortical thicknesses were also significantly reduced in CD patients, including in the insula, cingulate cortex, and other cortical regions (8). Bao et al. investigated abnormal activation in brain regions in $\mathrm{CD}$ patients using lowfrequency fluctuation amplitude (ALFF) and found that CD patients showed significantly higher ALFF values in the anterior cingulate cortex, insula, and precuneus compared with HCs (9). Furthermore, the different activation in regions between two groups related to the amygdala, hippocampus, insula, putamen, and cerebellar regions was observed during a high-stress task, suggesting altered habituation to stress in CD patients (10). A recent metaanalysis reported reduced resting-state brain connectivity in the paracentral lobule and cingulate gyrus, as well as reduced GMV in the medial frontal gyrus in CD patients (11). While responding to an uncertain condition, CD patients were observed to have increased regional activation in the cingulate cortex, insula, amygdala, thalamus, and hippocampus (12). The reduction of nodal betweenness centrality was also detected in the right insula and cuneus in CD patients through the building of topological properties of networks (13). Moreover, compared to female HCs, female CD patients have been shown to have increased functional connectivity (FC) between the left anterior insula and all 4 frontoparietal network nodes, with a reciprocal FC between the right posterior parietal cortex and the left anterior insula (14). As for topology networks, CD patients showed a reduction of nodal betweenness centrality in the right insula and cuneus (15). Moreover, Rubio et al. found that $C D$ patients had significantly stronger activations in the cingulate cortex, insula, amygdala, and thalamus than did controls in the context of uncertainty concerning upcoming experimentally induced rectal discomfort $(11,12)$. As can be seen from the above-mentioned studies, the insula figures frequently in CD-related neuroimaging studies.

As a limbic sensory area, the insula receives afferents from the dorsal thalamus and is mutually connected with the amygdala, along with several associated cortical areas (16). Insula dysfunction is now considered a core feature of anxiety, pain, and addiction (17). Multiple disturbances have been reported following lesions of the insula, which are implicated in autonomic function, somatosensory and multimodal perception, body awareness, the emotion of disgust, mood, and volitional behavior $(17,18)$. In recent years, tract-tracing studies have verified that the insula is associated with visceral sensations and is now known to receive interoceptive information from all over the body (19-21). Growing evidence has demonstrated the important role of the insula in inflammatory bowel disease (22). Therefore, investigating the potential abnormalities of insula circuits is essential for improving our understanding of CD.

In this study, we used the voxel-based morphometry (VBM) method to explore whether there were significant GMV differences in the insula between CD patients and HCs, and to investigate the whole-brain structural covariance patterns and seed-based FC of the insulae between the two groups. We then evaluated the possible relationships between clinical information and neuroimaging findings. We hypothesized that CD patients might have (I) abnormal morphology in the insula, (II) different structural covariance patterns, (III) altered FC of the insula compared to HCs; and (IV) correlations between clinical information and neuroimaging findings. We present the following article in accordance with the Materials Design Analysis Reporting (MDAR) checklist (available at https://dx.doi.org/10.21037/qims-21-509). 


\section{Methods}

\section{Ethics statement}

With the approval of the Ethics Committee of the Second Affiliated Hospital of Xi'an Jiaotong University (No. 2020081), CD patients were recruited from the Outpatient Clinic of Gastroenterology at the Second Affiliated Hospital of Xi'an Jiaotong University. Patients with a previous $\mathrm{CD}$ diagnosis were contacted by registered telephone to determine whether they were willing to participate in the experiment for a certain amount of monetary compensation, while HCs were recruited through advertisement. The recruited participants completed the test in batches within a month. A careful basic evaluation was performed for each subject. All the examinations were performed by the same professional gastroenterologist and an experienced psychiatrist in the Second Affiliated Hospital of Xi'an Jiaotong University. All experimental treatment steps performed in this study were in accordance with the Declaration of Helsinki (as revised in 2013) (23). The participants were informed of all experimental procedures and signed informed consent.

\section{Participants}

In the current study, 85 participants were recruited, including $45 \mathrm{CD}$ patients and $40 \mathrm{HCs}$. CD was assessed using the $\mathrm{CD}$ activity index (CDAI), $\mathrm{CD}$ endoscopic index of severity (CDEIS), and disease duration $(24,25)$. A visual analogue scale (VAS) was used to evaluate the pain index of patients. According to the VAS pain score, pain was divided into 11 grades from 0 to 10: 0 for no pain and 10 for excruciating pain. The final score was determined by dividing the sum of the daily pain scores by the number of days in pain. All participants underwent hospital anxiety and depression scale (HADS) evaluation. The HADS included two subscales, the HADS-A and HADS-D, which were used to quantify the levels of anxiety and depression of all participants. Both HADS-A and HADS-D consisted of 14 items, each with a score from 0 to 21 which indicated the following: 0 to 7 , normal; 8 to 10 , marginal; and 11 to 21 , abnormal.

The inclusion criteria of $\mathrm{CD}$ patients were as follows: (I) right handedness; (II) aged between 18 and 50; (III) education period $>6$ years; (IV) disease in remission over 12 months; (V) CDAI <150; (VI) CDEIS scores <3; and (VII) HADS-A and HADS-D scores $\leq 10$. All participants underwent conventional examination to exclude participants with metal implants and claustrophobia. The exclusion criteria for CD patients were as follows: (I) patients with metal implants; (II) patients with claustrophobia; (III) currently menstruating, pregnant, or lactating women; (IV) patients who underwent CD-related abdominal surgery; (V) those with a history of corticosteroids, antitumor necrosis factor alpha (anti-TNF- $\alpha$ ) agent, or psychotropic drug use in the past 3 months; and (VI) patients with a current or past history of psychiatric and neurological disorders, head trauma, or loss of consciousness.

The basic inclusion criteria of HCs were as follows: (I) right handedness; (II) aged between 18 and 50 years; (III) education period $>6$ years; (IV) HADS-A and HADS-D scores $\leq 10$; and $(\mathrm{V})$ no abdominal symptoms, intestinal transport disorders, or abdominal surgery history over the past 12 months. The HCs underwent similar basic evaluations as mentioned above. They had no history of familial psychiatric or neurological disorders and had refrained from alcohol or drug consumption in the week prior.

\section{Image acquisition}

The imaging data were acquired on a 3.0-T MR scanner (Signa HDXT, GE Healthcare) in the Department of Radiology at the Second Affiliated Hospital of Xi'an Jiaotong University. In order to diminish scanner noise and minimize head motion, all participants were instructed to lie in the supine position along a pair of foam pads. T1weighted images were obtained for each participant using a 3-dimensional spoiled gradient recall sequence with the following parameters: repetition time $(\mathrm{TR})=8.78 \mathrm{~ms}$, echo time $(\mathrm{TE})=2.83 \mathrm{~ms}$, field of view $(\mathrm{FOV})=240 \mathrm{~mm} \times 240 \mathrm{~mm}$, matrix size $=256 \times 256$, in-plane resolution $=0.94 \mathrm{~mm} \times$ $0.94 \mathrm{~mm}$, slice thickness $=1.0 \mathrm{~mm}$ (no gaps), flip angle (FA) $=12^{\circ}$, and slices $=150$. A single-shot gradient-recalled echo planar imaging sequence was then performed to acquire functional images for each participant, for which participants were instructed to relax, keep their eyes closed, stay awake, remain still, and to not to think about anything. The specific parameters were as follows: $\mathrm{TR}=2,000 \mathrm{~ms}, \mathrm{TE}=30 \mathrm{~ms}$, FOV $=240 \mathrm{~mm} \times 240 \mathrm{~mm}$, matrix size $=64 \times 64$, in-plane resolution $=3.75 \mathrm{~mm} \times 3.75 \mathrm{~mm}$, slice thickness $=3.5 \mathrm{~mm}$ (no gaps), $\mathrm{FA}=90^{\circ}$, and slices $=43$.

\section{Structural data preprocessing}

Structural image preprocessing was performed in a computational anatomy toolbox (CAT12) for SPM12 

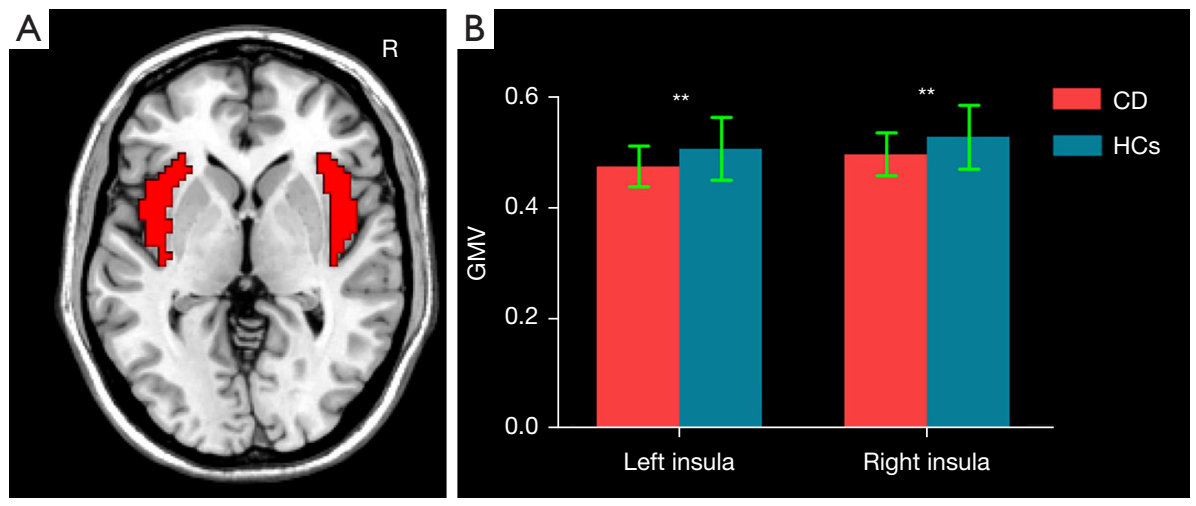

Figure 1 The mean GMV differences between CD patients and HCs within the bilateral insula. (A) The bilateral insula masks; (B) CD patients had significantly decreased GMV in the bilateral insula compared to HCs. ** $\mathrm{P}<0.01$. CD, Crohn's disease; HCs, healthy controls; GMV, gray matter volume.

(http://www.fil.ion.ucl.ac.uk/spm) run by MATLAB (MathWorks, Natick, MA, USA). The structural MRI images in the original space were transformed into Montreal Neurological Institute (MNI) 152 standard space by affine transformation to correct the brain position and rotation angle of different participants in the scanning process. Next, the images were divided into $1.5 \mathrm{~mm} \times 1.5 \mathrm{~mm} \times 1.5 \mathrm{~mm}$ GMV. To analyze and correct the volume difference of local GM, the segmented images were modulated by nonlinear transformation. Finally, to improve the signal-to-noise ratio and the impact of image registration error, the resulting GM images were smoothed with an isotropic Gaussian kernel of $3 \mathrm{~mm}$ full-width at half maximum (FWHM).

\section{Functional data preprocessing}

Functional images were processed using the Data Processing \& Analysis for Brain Imaging (DPABI) toolkit (version v4.3, http://rfmri.org/dpabi) under the MATLAB platform. Firstly, the first 5 time points were removed to ensure the stability of the initial signal. The remaining images underwent slice-timing correction to solve the problem of time delay between different slices. Head motion realignment was used to eliminate subjects with head-motion parameters that exceeded $2 \mathrm{~mm}$ translation and $2^{\circ}$ rotation. The corrected images were further spatially normalized to the MNI152 template using an echo-planar imaging (EPI) template and resampled to $3 \mathrm{~mm} \times 3 \mathrm{~mm} \times 3 \mathrm{~mm}$ volumes. The images were then smoothed with a Gaussian kernel with a FWHM of $6 \mathrm{~mm}$. Meanwhile, 24 head-motion parameters (6 head motion parameters, 6 head motion parameters 1 time point before, and the 12 corresponding squared items), cerebrospinal fluid (CSF), and white matter signal were used as nuisance covariates for regression. After detrending the time series, the filter with a bandwidth of $0.01-0.1 \mathrm{~Hz}$ was used to remove the interference of low-frequency drift and high-frequency physiological noise.

\section{Statistical analysis}

The chi-square test and 2-sample t test were used to analyze the demographic and clinical data, as well as the differences of the mean GMV of the insulae bilaterally between the two groups. These analyses were performed with SPSS 22.0 (IBM, Armonk, NY, USA). We set the significance level at $\mathrm{P}<0.05$.

The bilateral insulae in the automated anatomical labeling (AAL) template were selected as masks (Figure 1A). A group difference of voxel-based GMV between CD patients and HCs within the bilateral insula masks was examined using a 2 -sample $t$ test with the inclusion of age, sex, and total intracranial volume (TIV) as covariates $[\mathrm{P}<0.05$; cluster-level false discovery rate (FDR)]. VBM was subsequently reperformed with the inclusion of HADS-A and HADS-D as additional covariates.

Spheres with a radius of $3 \mathrm{~mm}$ centered on the peak T-value, which showed the significant GMV differences in the insular between CD patients and HCs, were taken as regions of interest (ROIs) to conduct the following structural covariance and FC analysis. Whole-brain structural covariance analyses were performed on the modulated GM images for each group using the general linear model in SPM12. The mean GMV of each ROI was included in the multiple regression models as a covariate 
Table 1 Clinical and demographic information of CD patients and healthy controls (HCs)

\begin{tabular}{lccc}
\hline \multirow{2}{*}{ Items } & \multicolumn{2}{c}{ Mean $\pm \mathrm{SD}$} & \multirow{2}{*}{ P value } \\
\cline { 2 - 3 } & CD patients & Healthy controls & \\
\hline Age (years) & $32.58 \pm 7.27$ & $30.68 \pm 5.78$ & $0.19^{\mathrm{a}}$ \\
Sex (male/female) & $25 / 20$ & $22 / 18$ & $1.00^{\mathrm{b}}$ \\
Weight (kg) & $57.28 \pm 8.11$ & $59.83 \pm 6.71$ & $0.12^{\mathrm{a}}$ \\
CDAl & $90.21 \pm 57.28$ & - & - \\
CDEIS & $1.21 \pm 0.85$ & - & - \\
VAS & $3.00 \pm 1.89$ & - & - \\
Disease duration & $77.69 \pm 53.19$ & - & - \\
(months) & & & $<0.0001^{\mathrm{a}}$ \\
HADS-A & $5.22 \pm 2.49$ & $3.25 \pm 1.98$ & $0.018^{\mathrm{a}}$ \\
HADS-D & $4.33 \pm 3.33$ & $2.95 \pm 1.82$ & - \\
\hline
\end{tabular}

${ }^{a}$, the $\mathrm{P}$ value was obtained by 2 -sample $t$ test; ${ }^{\mathrm{b}}$, the $\mathrm{P}$ value was obtained by chi-square test. CD, Crohn's disease; SD, standard deviation; CDAI, Crohn's disease activity index; VAS, visual analogue scale; HADS-A/-D, hospital anxiety and depression scale (anxiety)/(depression).

of interest to obtain the group level whole-brain voxelwise structural covariance of the ROIs. One-sample $t$ tests were performed to map the voxels that expressed a significant positive GM covariance with each ROI in each group with the inclusion of age, sex, and TIV as covariates. Since emotional factors, such as anxiety and depression, might exert their role in $\mathrm{CD}$, we conducted the structural covariance patterns of the insulae with age, sex, TIV, HADS-A, and HADS-D being included as additional covariates. The cluster-level family-wise error (FWE) was used for multiple testing corrections $(\mathrm{P}<0.05)(26)$.

According to previous studies (27-29), we performed between-group analyses of structural covariance patterns by calculating the slope differences. The analysis used a multiple classic linear regression:

$$
Y=\beta_{0}+\beta_{1} X+\beta_{2} \text { Group }+\beta_{3}(X \times \text { Group })+\beta_{4} \text { Age }+\beta_{5} \operatorname{Sex}+\varepsilon
$$

In equation $1, X$ represents a vector of averaged GMV of the insular subregion ROIs and $Y$ represents a vector of the GMV of each region showing group differences. Group is a vector with binary group labels (with 1 indicating CD patients and -1 indicating HCs). Age and Sex were considered nuisance variables. Since $\beta_{3}$ was seen to show significant group-by-GMV interaction [see Ge et al. (27) for more details], we chose the $t$ value of $\beta_{3}$ as the statistic to conduct a permutation test $(1,000$ times; $\mathrm{P}<0.05)$ and further used Bonferroni correction to test the results $(\mathrm{P}<0.05 / 15=0.003)$.

We extracted the mean blood-oxygen-level-dependent (BOLD) time series of each ROI from each participant. The correlations between the mean time series and time series of whole-brain voxels were computed, and these were then converted to $z$ scores using Fisher's r-to-z transformation to generate the individual level whole-brain $z$ score map of each participant. Group FC differences of the insulae between $\mathrm{CD}$ patients and HCs were examined using 2-sample $t$-tests with the inclusion of age and sex as covariates. Similarly, we also added the HADS-A and HADS-D scores as covariates $(\mathrm{P}<0.05$; cluster-level FDR).

Pearson's correlation analysis was then applied to detect relationships between the clinical symptoms (e.g., disease duration; and CDAI, CDEIS, and HADS scores), mean GMV within each ROI, and the FC value of the insulae with abnormal regions in $\mathrm{CD}$ patients $[\mathrm{P}<0.05$; Bonferroni correction (30)].

\section{Results}

\section{Demographics and clinical characteristics}

As shown in Table 1, there were no significantly statistical differences between the CD patients and HCs in age, sex, or weight $(\mathrm{P}>0.05)$. However, $\mathrm{CD}$ patients showed markedly higher HADS-A and HADS-D scores $(\mathrm{P}<0.05)$.

\section{GMV results}

Compared with HCs, CD patients exhibited decreased GMV of the bilateral insula (left insula: $0.475 \pm 0.037 \mathrm{~mL}$ in CD patients $v s .0 .507 \pm 0.057 \mathrm{~mL}$ in $\mathrm{HCs}$; $\mathrm{P}=0.007$; right insula: $0.496 \pm 0.039 \mathrm{~mL}$ in CD patients vs. $0.528 \pm 0.057 \mathrm{~mL}$ in HCs, $\mathrm{P}=0.009$ ) (Figure 1B). Figure 2 and Table 2 display the significant GMV differences of the insulae between the two groups. CD patients showed significantly decreased GMVs in the left dorsal anterior insula (dAI) and bilateral posterior insula (PI) compared with HCs. The inclusion of anxiety and depression made the GMV difference in the left dAI nonsignificant. Taking the three regions as ROIs, the correlation analysis demonstrated that there was no significant correlation between the GMVs of ROIs and disease duration, CDAI, CDEIS, or HADS scores in CD patients. 


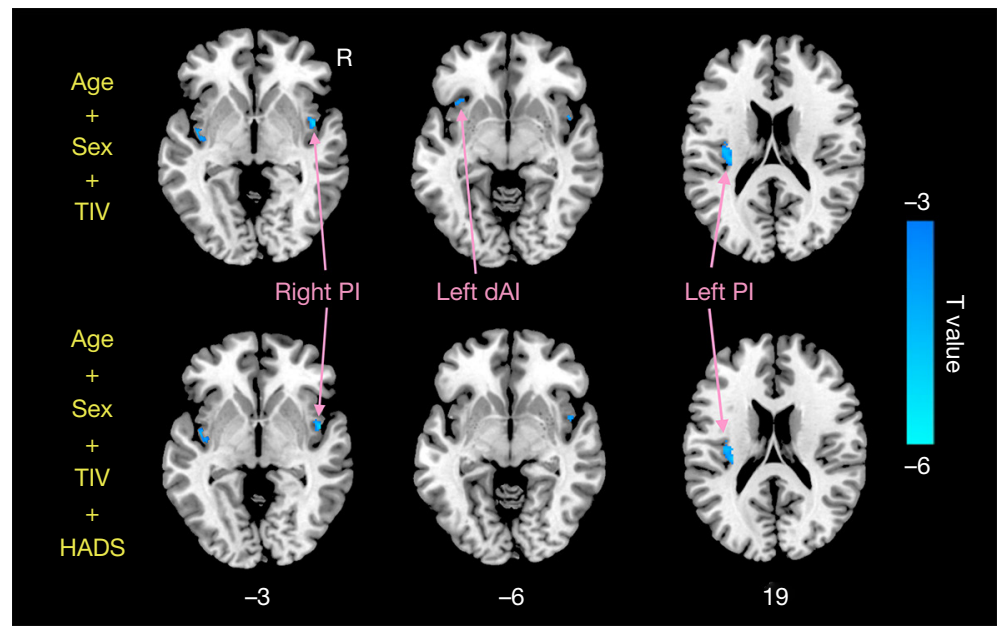

Figure 2 Significant differences between the GMV of CD patients and HCs. The top row shows the differences with age, sex, and TIV as covariates, and the bottom row shows the differences with age, sex, TIV, and HADS as covariates. CD, Crohn's disease; HCs, healthy controls; GMV, gray matter volume; TIV, total intracranial volume; HADS, hospital anxiety and depression scale; dAI, dorsal anterior insula; PI, posterior insula.

Table 2 Subregions of the insula showing significant GMV differences between the CD patients and healthy controls (HCs)

\begin{tabular}{lccccc}
\hline \multirow{2}{*}{ Regions } & Hem & \multicolumn{4}{c}{ MNI } \\
\cline { 3 - 5 } & & $\mathrm{x}$ & $\mathrm{y}$ & $\mathrm{z}$ & T value \\
\hline $\mathrm{dAl}$ & $\mathrm{L}$ & -33 & 21 & -6 & -3.87 \\
$\mathrm{PI}$ & $\mathrm{R}$ & 48 & 3 & -3 & -5.11 \\
$\mathrm{PI}$ & $\mathrm{L}$ & -36 & -18 & 18 & -4.23 \\
\hline
\end{tabular}

GMV, gray matter volume; MNI, Montreal Neurological Institute; BA, Brodmann area; Hem, hemisphere; L, left; R, right; dAI, dorsal anterior insula; PI, posterior insula.

\section{Structural covariance results}

Figures 3,4 and Table 3 show the different patterns of structural covariance in the two groups associated with the 3 insula subregions. There was an increased association between the $\mathrm{dAI}$ and regions, including the caudate, dorsal posterior cingulate cortex (dPCC), and parahippocampus/ hippocampus (paraHIPP/HIPP), as well as a decreased association between the dAI and precuneus in the CD patients compared to the HCs. The supplementary motor area (SMA), anterior middle cingulate cortex (aMCC), dorsolateral prefrontal cortex (dlPFC), amygdala, and paraHIPP/HIPP showed decreased covariance with the right $\mathrm{PI}$ in the $\mathrm{CD}$ patients compared to HCs. For the left PI ROI, decreased structural covariance was observed between the right PI and regions including the precuneus, dPCC, angular gyri, and putamen. The inclusion of anxiety and depression as covariates (with age and sex) did not lead to obvious changes in the structural covariance patterns of CD patients (Figure 3).

After interaction linear model analysis, the structural covariance differences of the insular subregions were still found to be significantly related to the bilateral precuneus, angular gyri, bilateral putamen, and SMA (Figure 4).

\section{FC results}

For the left dAI ROI, CD patients had markedly decreased FCs with the posterior MCC (pMCC), SMA, caudate, paraHIPP/HIPP, and anterior cingulate cortex (ACC) compared with HCs. For the right PI ROI, CD patients exhibited decreased FC with the aMCC, dIPFC, amygdala, and paraHIPP/HIPP compared with HCs. As for the left PI, CD patients showed decreased FC with the primary somatosensory (S1), SMA, and aMCC (Figure 5 and Table 4). When anxiety and depression were employed as covariates (with age and sex), we found that several FC differences were not significant, and were involved in connectivity between the left dAI and right ACC. Also, FC differences between the right PI and regions including bilateral aMCC, right dlPFC, and left amygdala were not significant (Figure 5). 

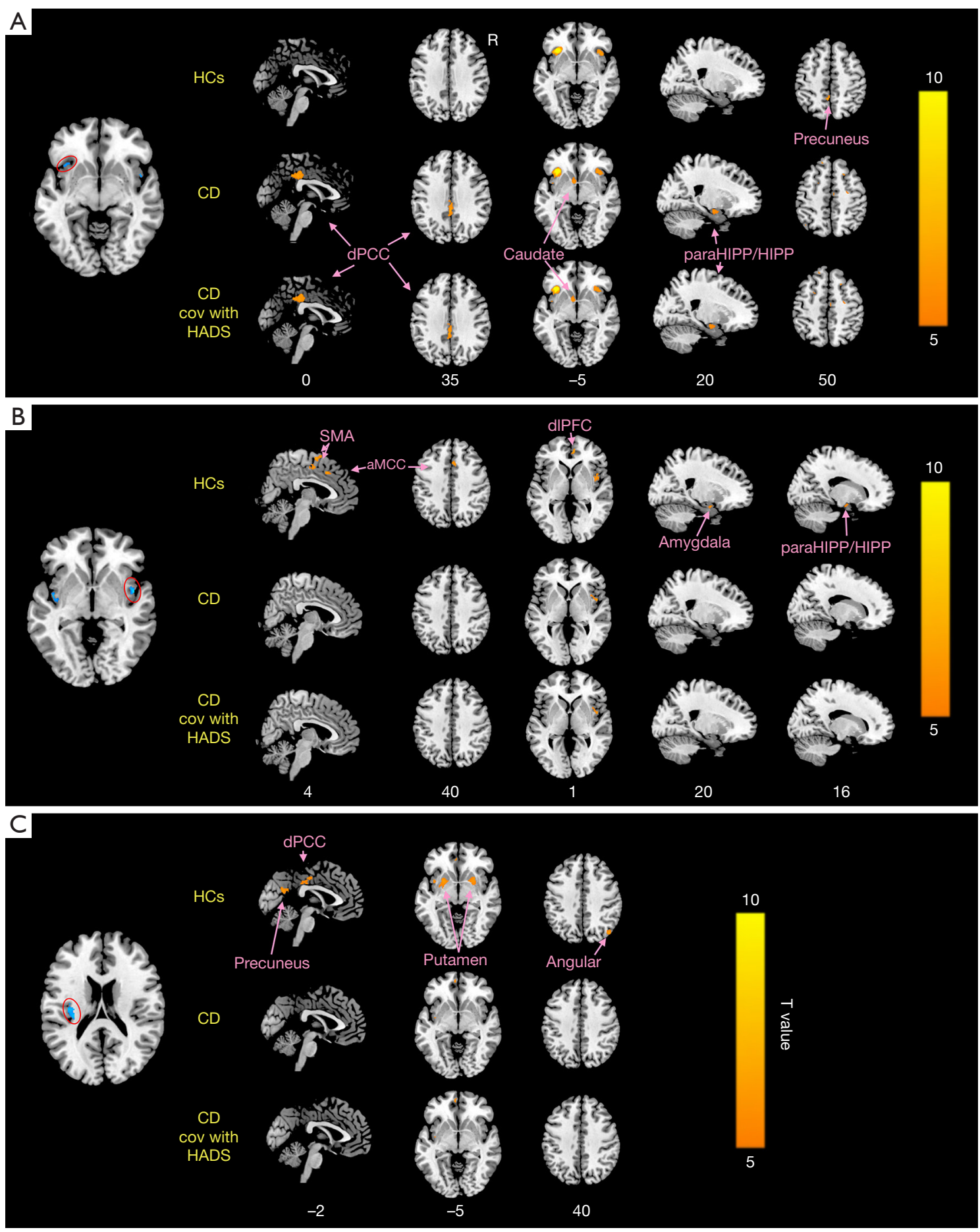

Figure 3 The seed-based structural covariance patterns in CD patients and HCs. The structural covariance patterns related with the left dAI (A), the right PI (B), and the left PI (C) seed in the two groups. CD, Crohn's disease; HCs, healthy controls; ROI, region of interest; dAI, dorsal anterior insula; PI, posterior insula; dPCC, dorsal posterior cingulate cortex; aMCC, anterior middle cingulate cortex; paraHIPP/ HIPP, parahippocampus/hippocampus; SMA, supplementary motor area; dlPFC, dorsolateral prefrontal cortex. 

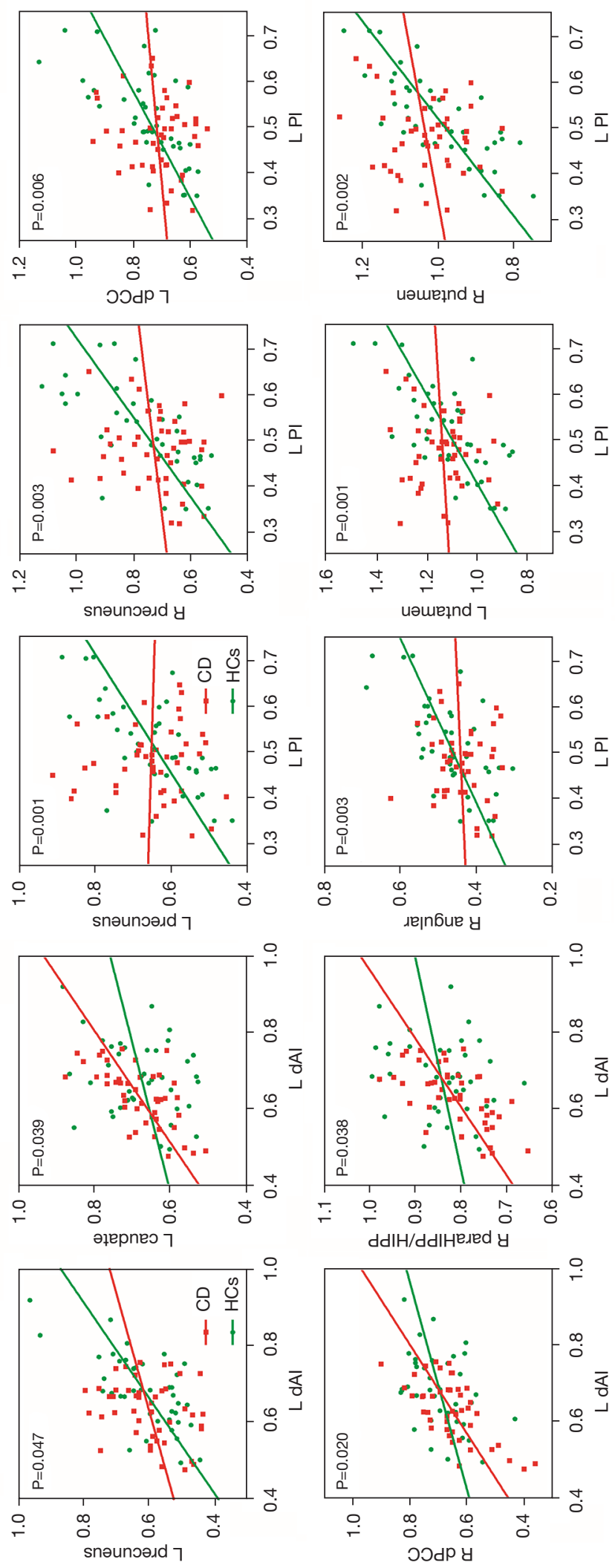
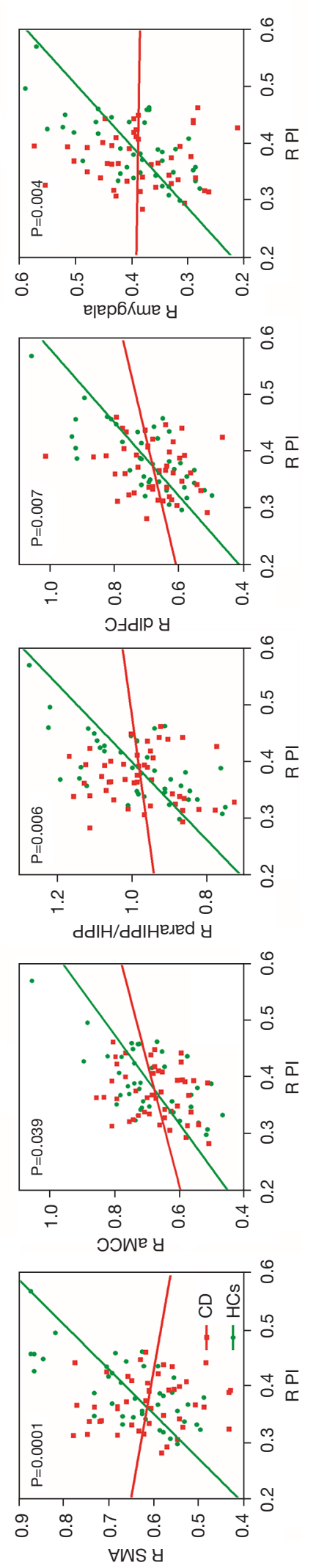

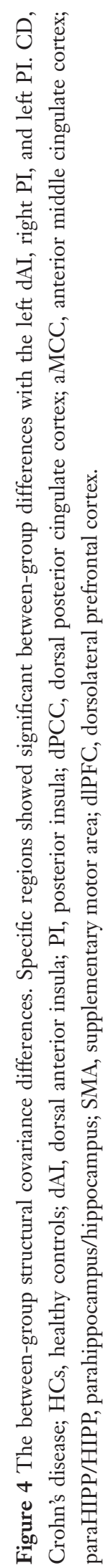


Table 3 Brain regions showed significant differences in seed-based covariance patterns with ROIs between CD patients and healthy controls (HCs)

\begin{tabular}{|c|c|c|c|c|c|c|c|c|c|c|c|c|c|}
\hline Regions & BA & \multicolumn{6}{|c|}{$\mathrm{HCs}$} & \multicolumn{6}{|c|}{$C D$} \\
\hline \multicolumn{14}{|l|}{ Left dAl } \\
\hline Precuneus & 23 & $L$ & -3 & -48 & 54 & 5.86 & 96 & & & & & & \\
\hline Caudate & 25 & & & & & & & L & -6 & 9 & -9 & 5.97 & 147 \\
\hline paraHIPP/HIPP & 35 & & & & & & & $\mathrm{R}$ & 21 & -9 & -18 & 5.89 & 169 \\
\hline \multicolumn{14}{|l|}{ Right PI } \\
\hline SMA & 6 & $\mathrm{R}$ & 6 & 0 & 66 & 7.62 & 210 & & & & & & \\
\hline aMCC & 24 & $\mathrm{R}$ & 3 & 18 & 39 & 6.54 & 141 & & & & & & \\
\hline paraHIPP/HIPP & 34 & $\mathrm{R}$ & 21 & 0 & -21 & 5.35 & 88 & & & & & & \\
\hline \multicolumn{14}{|l|}{ Left PI } \\
\hline \multirow[t]{2}{*}{ Precuneus } & 23 & $\mathrm{~L}$ & -3 & -60 & 24 & 6.27 & 204 & & & & & & \\
\hline & & $\mathrm{R}$ & 3 & -48 & 21 & 5.66 & 217 & & & & & & \\
\hline $\mathrm{dPCC}$ & 23 & $\mathrm{~L}$ & -3 & -18 & 45 & 5.55 & 221 & & & & & & \\
\hline Angular & 39 & $\mathrm{R}$ & 48 & -69 & 39 & 5.91 & 99 & & & & & & \\
\hline \multirow[t]{2}{*}{ Putamen } & 48 & $L$ & 27 & 9 & 3 & 6.87 & 400 & & & & & & \\
\hline & & $\mathrm{R}$ & -24 & 9 & 3 & 7.04 & 303 & & & & & & \\
\hline
\end{tabular}

ROls, regions of interest; BA, Brodmann area; Hem, hemisphere; L, left; R, right; dAl, dorsal anterior insula; PI posterior insula; dPCC, dorsal posterior cingulate cortex; paraHIPP/HIPP, parahippocampus/hippocampus; SMA, supplementary motor area; aMCC, anterior middle cingulate cortex; dIPFC, dorsolateral prefrontal cortex.

\section{Correlation results}

CD patients exhibited significant negative correlations between the CDAI and FC of the bilateral paraHIPP/HIPP and the left dAI and right PI (Figure 6).

\section{Discussion}

In this study, we investigated the altered structural covariance and $\mathrm{FC}$ of the insula in $\mathrm{CD}$ patients, and the main findings are described below. (I) Compared to HCs, CD patients exhibited higher HADS scores and decreased GMV in the bilateral insula. (II) CD patients showed significantly decreased GMVs in the insular subregions related to the left $\mathrm{dAI}$ and bilateral PI, which were selected as ROIs, while the inclusion of anxiety and depression made the difference of GMV in the left dAI nonsignificant. (III)
For the dAI ROI, CD patients showed increased structural association with the caudate, dPCC, and paraHIPP/ HIPP, as well as decreased structural association with the precuneus. For the PI ROIs, CD patients showed decreased structural association with regions including the SMA, aMCC, dIPFC, amygdala, paraHIPP/HIPP, precuneus, dPCC, angular gyri, and putamen. (IV) The insula-related FC differences were mainly located in the MCC, SMA, dlPFC, caudate, ACC, S1, amygdala, and paraHIPP/ HIPP. (V) With anxiety and depression as covariates, the significant FC differences between the left dAI and ACC, as well as between the right PI and AlPFC, ACC, and amygdala disappeared in CD patients. (VI) There were negative correlations between the CDAI and FC of the bilateral paraHIPP/HIPP with the left dAI and right PI.

The insula, which is located in the interface of the cognitive, homeostatic, and affective systems of the human 

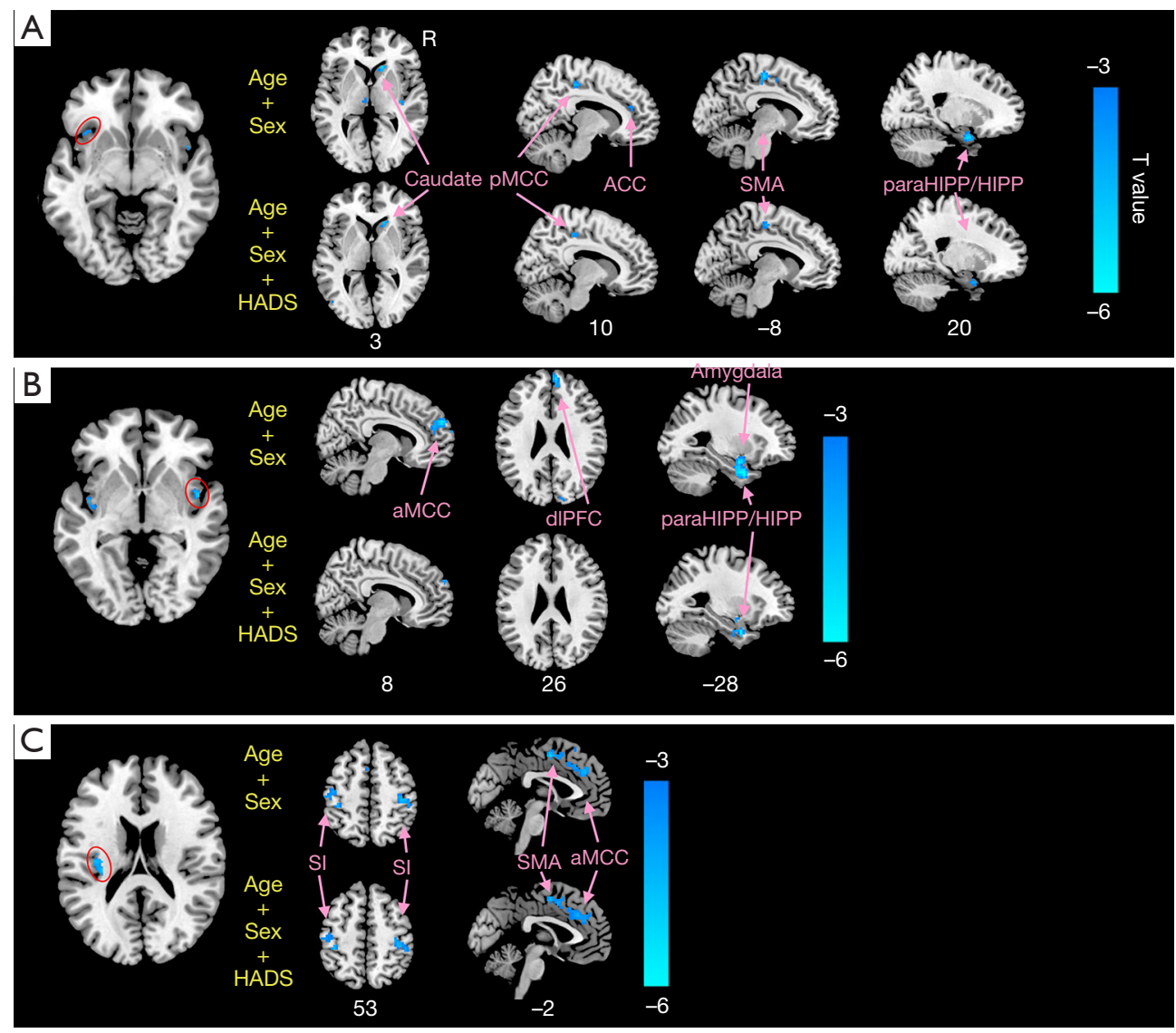

Figure 5 Results of functional connectivity. The brain regions showing significantly decreased functional connectivity with the left dAI (A), the right PI (B), and left PI (C) in CD patients compared to HCs. CD, Crohn's disease; HCs, healthy controls; dAI, dorsal anterior insula; PI posterior insula; pMCC/aMCC, posterior/anterior middle cingulate cortex; paraHIPP/HIPP, parahippocampus/hippocampus; ACC, anterior cingulate cortex; dlPFC, dorsolateral prefrontal cortex; S1, primary somatosensory; SMA, supplementary motor area.

brain, provides a link between stimulus-driven processing and brain areas involved in monitoring the internal environment (31). Bao et al. demonstrated that there was a marked decrease in the GMV of the bilateral insula of CD patients (8). The changed GMV in the insula were included in our current results. According to the divided insula subregions in previous studies (32,33), we found significantly decreased GMV in the left dAI and bilateral PI.

Intercessory information about the overall physiological condition of the body is received by the PI and projected to the AI for subjective assessment of the internal condition (33). Specifically, the AI is thought to help identify the emotional state hidden under visceral sensation and to interact with higher-order brain regions, thus subjectively evaluating the social and emotional interaction between individuals and the environment. Meanwhile, the PI is considered to receive emotional inputs from the thalamus, limbic, and brainstem structures, and to help with recognizing internal changes within the body (31). A reduced local connectivity in this region could be a marker of disturbed abdominal sensory perception and of altered emotional evaluation of these stimuli in CD patients (15). Therefore, the decreased GMV in the subregions of the insula might be associated with brain dysfunctions of sensory transmission and emotional processing in $\mathrm{CD}$ patients.

MCC abnormalities were observed in our results. The MCC has two subdivisions: the anterior MCC (aMCC) and the posterior MCC (pMCC). The aMCC is critical for feedback-mediated decision-making and has key outputs to the supplementary motor area/pre-supplementary motor area and spinal cord, while the pMCC is involved in the multisensory orientation of the head and body in space (34-36). Taylor et al. proposed that the system linking the entire insula with the pMCC could be a general salience 
Table 4 Brain regions showing significantly decreased functional connectivity with ROIs between CD patients and healthy controls (HCs)

\begin{tabular}{|c|c|c|c|c|c|c|c|}
\hline \multirow{2}{*}{ Regions } & \multirow{2}{*}{ BA } & \multirow{2}{*}{ Hem } & \multicolumn{3}{|c|}{ MNI } & \multirow{2}{*}{$\mathrm{T}$ value } & \multirow{2}{*}{ Voxels } \\
\hline & & & $x$ & y & $z$ & & \\
\hline \multicolumn{8}{|l|}{ Left dAl } \\
\hline pMCC & 23 & $\mathrm{R}$ & 9 & -30 & 48 & -3.73 & 57 \\
\hline SMA & 25 & $\mathrm{R}$ & 15 & 24 & 3 & -4.35 & 60 \\
\hline paraHIPP/HIPP & 32 & $\mathrm{R}$ & 9 & 39 & 21 & -3.23 & 93 \\
\hline ACC & 23 & $\mathrm{R}$ & 9 & -30 & 48 & -3.73 & 55 \\
\hline \multicolumn{8}{|l|}{ Right PI } \\
\hline aMCC & 32 & $\mathrm{R}$ & 6 & 48 & 24 & -3.94 & 83 \\
\hline Amygdala & 34 & $L$ & -27 & -3 & -12 & -4.43 & 35 \\
\hline \multirow[t]{2}{*}{ paraHIPP/HIPP } & 28 & $\mathrm{~L}$ & -27 & 3 & -27 & -5.48 & 72 \\
\hline & & $\mathrm{R}$ & 21 & 3 & -27 & -5.04 & 92 \\
\hline \multicolumn{8}{|l|}{ Left PI } \\
\hline \multirow[t]{2}{*}{ S1 } & 2,3 & $\mathrm{~L}$ & -45 & -24 & 57 & -3.62 & 103 \\
\hline & & $\mathrm{R}$ & 39 & -24 & 51 & -4.50 & 111 \\
\hline SMA & 6 & $L$ & -3 & -3 & 51 & -4.12 & 117 \\
\hline aMCC & 24 & L & -3 & -6 & 51 & -4.31 & 65 \\
\hline
\end{tabular}

ROls, regions of interest; BA, Brodmann area; MNI, Montreal Neurological Institute; Hem, hemisphere; L, left; R, right; dAI, dorsal anterior insula; PI posterior insula; pMCC/aMCC, posterior/anterior middle cingulate cortex; SMA, supplementary motor area; paraHIPP/HIPP, parahippocampus/hippocampus; ACC, anterior cingulate cortex; dIPFC, dorsolateral prefrontal cortex; S1, primary somatosensory.
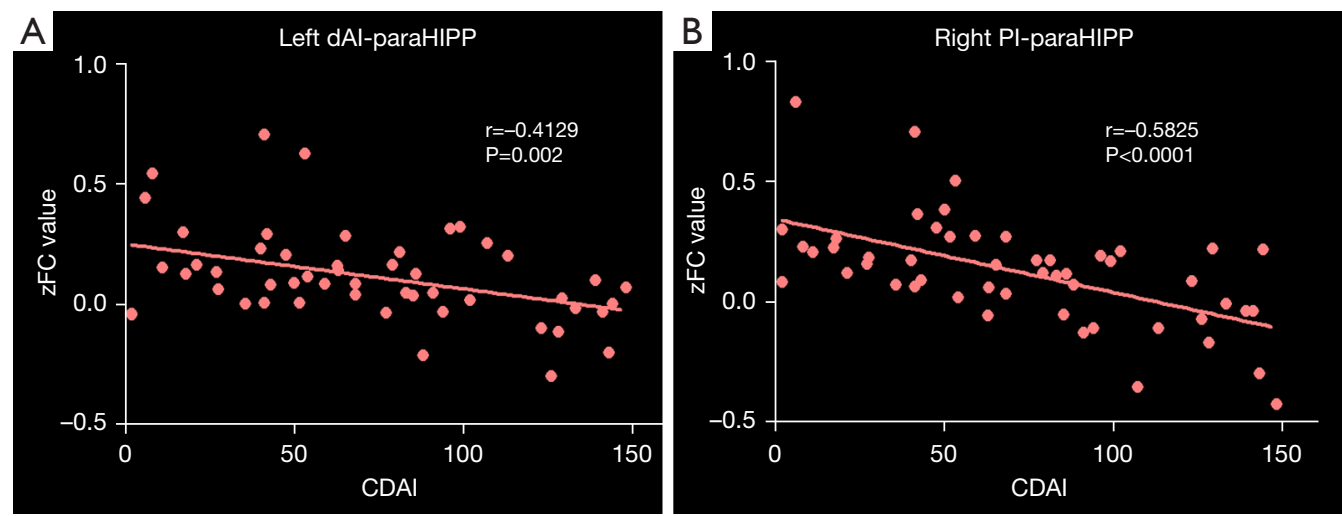

Figure 6 Correlation results. Negative correlation between the CDAI and altered functional connectivity of the left dAI with paraHIPP/ HIPP (A), and the right PI with paraHIPP/HIPP (B). CDAI, Crohn's disease activity index; dAI, dorsal anterior insula; PI posterior insula; paraHIPP/HIPP, parahippocampus/hippocampus. 
and action system (33). A study of mice revealed that the MCC was responsible for inducing and maintaining pain hypersensitivity, even in the absence of pain stimuli, and worked with the posterior insula to modulate chronic pain $(11,37)$. Accordingly, we postulate that MCC abnormalities may indicate impaired sensory stimulus localization and abnormal chronic pain regulation in CD patients.

We found abnormal structural covariance and $\mathrm{FC}$ of 3 ROIs with several regions in the default mode network $(\mathrm{DMN})$. The $\mathrm{DMN}$ refers to a set of brain regions that show greater metabolic activity during the resting state compared to other brain regions (38). The dominant functional characterization of the PCC arises out of its central role within the DMN (39). The dPCC shows strong connectivity with the rest of the DMN, but is also functionally highly connected to the frontoparietal networks of attention and executive control, suggesting a more prominent role of dPCC in cognitive control $(39,40)$. A recent study reported increased activity of the dPCC (specifically area d23) during evaluative feedback concerning the correctness of a decision, demonstrating the dorsal PCC to be involved in the behavioral adaptation to information from the environment (41).

As a key node of the DMN, the dlPFC is implicated in cognitive, affective, and sensory processing (42). Metaanalyses of functional brain imaging studies about responses to aversive rectal distension reported the activation of the bilateral AI and the left dIPFC in HCs (43). Additionally, a recent study indicated that the correlation of activity in AI with both perceived intensity and unpleasantness could be reduced by the right dIPFC and proposed that this dIPFC can actively control pain perception by regulating corticalsubcortical and cortical-cortical pathways (44).

As a part of the DMN, the angular gyri are linked to memory retrieval and are regarded as brain scene buffers (45). Laird et al. proposed that the bilateral angular gyri that act as dynamic self-referential regions during rest are associated with the significant behavioral features of interoception and somesthesis (46). Alternatively, the angular gyrus was reported to be associated with self-related processing and cognitive behavior regulation, and essential in fighting anxiety and negative emotions $(47,48)$.

Among the regions of the DMN, the precuneus stands out by virtue of its distinctive role. One study showed that the precuneus was involved in self-processing tasks and was associated with activated self-awareness in patients with chronic pain, which might be related to how these patients perceive their relationship with the outside world (49).
Moreover, during pain expectation, the precuneus has been shown to exhibit decreased activation associated with expected pain intensity, suggesting its involvement in the perception of impending pain (50). Based on the above research findings, the abnormal structural covariance pattern and FC of insula with nodes in the DMN may indicate reduced self-relevant sensation assessment ability in response to visceral stimulation and behavioral adaptation to information from the environment in CD patients.

Our results showed abnormal structural covariance and FC of 3 ROIs with specific regions related to the sensorimotor network. The sensorimotor network receives sensory input from the surroundings and plays an important role in the body's sensory perception and the generation of appropriate motor responses (51). It has been reported that the primary motor cortex (M1) receives somatosensory input predominantly via $\mathrm{S} 1$, which explains why motorrelated deficits can arise from damage to $\mathrm{S} 1(52,53)$. It has also been reported that $\mathrm{S} 1$ is specifically sensitive to changes in incoming non-nociceptive input (54). The caudate, when combined with the putamen, is referred to as the striatum and is often considered jointly in function, which is the major input source of the basal ganglia $(55,56)$. The basal ganglia are implicated in sensation. Signals from olfactory, auditory and somatosensory inputs indicate that this structure can integrate multiple sensory modes and coordinate behavioral responses (57). It was found that the anterior insula downregulates the pain network through the caudate nucleus with extensive functional connections between the caudate and anterior insula during pain stimulation $(18,58)$. The SMA is considered to support a flexible engagement of sensorimotor processes. In the motor network, the SMA is responsible for movement coordination and thus belongs to the frontomesial cortex, which is anterior to the M1 $(59,60)$. Based on the above evidence, it can be speculated that the abnormal structural covariance and $\mathrm{FC}$ of insulae with the sensorimotor regions, including the S1, caudate, putamen, and SMA may impact the integration of poorly localized internal sensations with other sensory input to some extent, and may be important in planning a movement response.

As a major hub of the salience network, the AI serves to identify salient stimuli information and forward this information to the higher cognitive regions, thereby subjectively assessing the social and emotional interaction between individuals and the environment $(31,61)$. Interestingly, our results showed that the significant GMV difference in the AI disappeared with the inclusion of 
anxiety and depression as covariates. These findings suggest that the $\mathrm{AI}$ is preferentially involved in the transmission and processing of negative emotions. The amygdala is well known to play a critical role in emotional processing (18). For example, increased amygdala reactivity during processing of certain types of emotional stimuli has been noted in several anxiety disorders $(62,63)$. Moreover, recent studies $(64,65)$ have discovered that higher scores on several measures assessing anxiety proneness were associated with greater activation of the left amygdala and bilateral anterior insula, which is also consistent with our findings showing that CD patients had more severe anxiety and depression in this study. The ACC receives information from its topologically nearby orbitofrontal cortex and the amygdala, and projects the information to several regions, including the autonomic areas in the brainstem, insula, MCC, and striatum (66). Affective responses also extend to the adjacent aMCC (67). Neuroimaging evidence is described that the anterior limbic system including the orbitofrontal cortex and amygdala are involved in emotion and reward valuation, with the value representations being transmitted to the ACC and aMCC for action-outcome learning $(34,68)$. The caudal part of the pACC, which is close to the aMCC, is involved in the production of emotional expressions (67). As mentioned in the discussion above, the dlPFC is implicated in cognitive, affective, and sensory processing. In the context of emotion, an integrative review regarding the PFC suggested that IPFC might be a key component of the neural circuitry involved in the type of appraisals that initiate forms of controlled emotional regulation (69). In HCs, the dlPFC was found to be activated during anxiety induction, and the intensity of this activation was negatively correlated with anxiety, suggesting that poor dIPFC activation was associated with less downregulation of anxiety (70). In addition, with the inclusion of anxiety and depression as covariates, the dAI-related FC with the ACC, and the PI-related FC with the amygdala, aMCC and dlPFC were not significantly different between two groups. The disappearance of the FC patterns mentioned above illustrated the roles of these regions in the processing of negative emotions. Therefore, this study suggests that the decreased AI-related FC with the ACC, the PI-related FC with the amygdala, the ACC, and the dlPFC may affect negative emotional processing capability, reflecting the high sensitivity to negative emotions and decreased inhibitory control over the visceral aversive sensory processing in CD patients.

\section{Limitations}

There are still several limitations that need to be considered when interpreting the present findings. Although we speculated that changes in pain-related brain regions were associated with clinical symptoms of CD, there was a lack of conclusive evidence, such as further analysis of VAS scores as covariates. It is necessary to further systematically study the relationship between pain and neuroimaging. Functional networks can provide coactivated or coinactivated connectivity patterns between different brain regions. Therefore, the study of functional networks or joint analysis of functional and structural networks could provide more imaging evidence for $\mathrm{CD}$.

\section{Conclusions}

In this study, we detected the decreased GMV in the subregions of the insula, identified the aberrant patterns of structural covariance and FC based on the ROIs, and discovered a negative correlation between the CDAI and FC of ROIs and specific regions. These structural covariance/FC abnormalities of the insula may be associated with prominent external stimuli and visceral afferent pathway injuries, and may be implicated in high sensitivity in negative emotion, decreased inhibitory control over the processing of visceral pain, and dysfunction of assessing self-relevant sensations in CD patients. Our findings may provide new neuroimaging evidence in the study of $\mathrm{CD}$.

\section{Acknowledgments}

Funding: This study was financially supported by the National Natural Science Foundation of China (No. 81771918) and the Shaanxi National Science Foundation (No. 2020JM-197).

\section{Footnote}

Reporting Checklist: The authors have completed the Materials Design Analysis Reporting (MDAR) checklist. Available at https://dx.doi.org/10.21037/qims-21-509

Conflicts of Interest: All authors have completed the ICMJE uniform disclosure form (available at https://dx.doi. org/10.21037/qims-21-509). The authors have no conflicts of interest to declare. 
Ethical Statement: The authors are accountable for all aspects of the work in ensuring that questions related to the accuracy or integrity of any part of the work are appropriately investigated and resolved. The study was conducted in accordance with the Declaration of Helsinki (as revised in 2013). The Ethics Committee of the Second Affiliated Hospital of Xi'an Jiaotong University in China approved all of the procedures of this study (No. 2020081). All participants were informed of all experimental procedures related to the experiment and signed informed consent prior to the experiment.

Open Access Statement: This is an Open Access article distributed in accordance with the Creative Commons Attribution-NonCommercial-NoDerivs 4.0 International License (CC BY-NC-ND 4.0), which permits the noncommercial replication and distribution of the article with the strict proviso that no changes or edits are made and the original work is properly cited (including links to both the formal publication through the relevant DOI and the license). See: https://creativecommons.org/ licenses/by-nc-nd/4.0/.

\section{References}

1. Torres J, Mehandru S, Colombel JF, Peyrin-Biroulet L. Crohn's disease. Lancet 2017;389:1741-55.

2. Hovde $\varnothing$, Kempski-Monstad I, Småstuen MC, Solberg IC, Henriksen M, Jahnsen J, Stray N, Moum BA. Mortality and causes of death in Crohn's disease: results from 20 years of follow-up in the IBSEN study. Gut 2014;63:771-5.

3. Gracie DJ, Hamlin PJ, Ford AC. The influence of the brain-gut axis in inflammatory bowel disease and possible implications for treatment. Lancet Gastroenterol Hepatol 2019;4:632-42.

4. Mayer EA, Labus JS, Tillisch K, Cole SW, Baldi P. Towards a systems view of IBS. Nat Rev Gastroenterol Hepatol 2015;12:592-605.

5. Bonaz BL, Bernstein CN. Brain-gut interactions in inflammatory bowel disease. Gastroenterology 2013;144:36-49.

6. Mayer EA, Naliboff BD, Craig AD. Neuroimaging of the brain-gut axis: from basic understanding to treatment of functional GI disorders. Gastroenterology 2006;131:1925-42.

7. Thomann AK, Schmitgen MM, Kmuche D, Ebert MP, Thomann PA, Szabo K, Gass A, Griebe M, Reindl W,
Wolf RC. Exploring joint patterns of brain structure and function in inflammatory bowel diseases using multimodal data fusion. Neurogastroenterol Motil 2021;33:e14078.

8. Bao CH, Liu P, Liu HR, Wu LY, Shi Y, Chen WF, Qin W, Lu Y, Zhang JY, Jin XM, Wang XM, Zhao JM, Liu $\mathrm{XM}$, Tian J, Wu HG. Alterations in brain grey matter structures in patients with crohn's disease and their correlation with psychological distress. J Crohns Colitis 2015;9:532-40.

9. Bao C, Liu P, Liu H, Jin X, Shi Y, Wu L, Zeng X, Zhang J, Wang D, Calhoun VD, Tian J, Wu H. Difference in regional neural fluctuations and functional connectivity in Crohn's disease: a resting-state functional MRI study. Brain Imaging Behav 2018;12:1795-803.

10. Agostini A, Filippini N, Benuzzi F, Bertani A, Scarcelli A, Leoni C, Farinelli V, Riso D, Tambasco R, Calabrese C, Rizzello F, Gionchetti P, Ercolani M, Nichelli P, Campieri $M$. Functional magnetic resonance imaging study reveals differences in the habituation to psychological stress in patients with Crohn's disease versus healthy controls. J Behav Med 2013;36:477-87.

11. Yeung AWK. Structural and functional changes in the brain of patients with Crohn's disease: an activation likelihood estimation meta-analysis. Brain Imaging Behav 2021;15:807-18.

12. Rubio A, Pellissier S, Van Oudenhove L, Ly HG, Dupont P, Tack J, Dantzer C, Delon-Martin C, Bonaz B. Brain responses to uncertainty about upcoming rectal discomfort in quiescent Crohn's disease - a fMRI study. Neurogastroenterol Motil 2016;28:1419-32.

13. Liu P, Li R, Bao C, Wei Y, Fan Y, Liu Y, Wang G, Wu H, Qin W. Altered topological patterns of brain functional networks in Crohn's disease. Brain Imaging Behav 2018;12:1466-78.

14. Kornelsen J, Wilson A, Labus JS, Witges K, Mayer EA, Bernstein CN. Brain Resting-State Network Alterations Associated With Crohn's Disease. Front Neurol 2020;11:48.

15. Thomann AK, Reindl W, Wüstenberg T, Kmuche D, Ebert MP, Szabo K, Wolf RC, Hirjak D, Niesler B, Griebe M, Thomann PA. Aberrant brain structural large-scale connectome in Crohn's disease. Neurogastroenterol Motil 2019;31:e13593.

16. Nieuwenhuys R. The insular cortex: a review. Prog Brain Res 2012;195:123-63.

17. Ibañez A, Gleichgerrcht E, Manes F. Clinical effects of insular damage in humans. Brain Struct Funct 
2010;214:397-410.

18. Menon V. Large-scale brain networks and psychopathology: a unifying triple network model. Trends Cogn Sci 2011;15:483-506.

19. Mufson EJ, Mesulam MM, Pandya DN. Insular interconnections with the amygdala in the rhesus monkey. Neuroscience 1981;6:1231-48.

20. Jakab A, Molnár PP, Bogner P, Béres M, Berényi EL. Connectivity-based parcellation reveals interhemispheric differences in the insula. Brain Topogr 2012;25:264-71.

21. Cloutman LL, Binney RJ, Drakesmith M, Parker GJ, Lambon Ralph MA. The variation of function across the human insula mirrors its patterns of structural connectivity: evidence from in vivo probabilistic tractography. Neuroimage 2012;59:3514-21.

22. Hong JY, Labus JS, Jiang Z, Ashe-Mcnalley C, Dinov I, Gupta A, Shi Y, Stains J, Heendeniya N, Smith SR, Tillisch K, Mayer EA. Regional neuroplastic brain changes in patients with chronic inflammatory and noninflammatory visceral pain. PLoS One 2014;9:e84564.

23. World Medical Association. World Medical Association Declaration of Helsinki: ethical principles for medical research involving human subjects. JAMA 2013;310:2191-4.

24. Best WR, Becktel JM, Singleton JW. Rederived values of the eight coefficients of the Crohn's Disease Activity Index (CDAI). Gastroenterology 1979;77:843-6.

25. Mary JY, Modigliani R. Development and validation of an endoscopic index of the severity for Crohn's disease: a prospective multicentre study. Groupe d'Etudes Thérapeutiques des Affections Inflammatoires du Tube Digestif (GETAID). Gut 1989;30:983-9.

26. Loop MS, McClure LA. Testing for clustering at many ranges inflates family-wise error rate (FWE). Int J Health Geogr 2015;14:4.

27. Ge R, Hassel S, Arnott SR, Davis AD, Harris JK, Zamyadi M, Milev R, Frey BN, Strother SC, Müller DJ, Rotzinger S, MacQueen GM, Kennedy SH, Lam RW, VilaRodriguez F. Structural covariance pattern abnormalities of insula in major depressive disorder: A CAN-BIND study report. Prog Neuropsychopharmacol Biol Psychiatry 2021;111:110194.

28. Wang X, Yu Y, Zhao W, Li Q, Li X, Li S, Yin C, Han Y. Altered Whole-Brain Structural Covariance of the Hippocampal Subfields in Subcortical Vascular Mild Cognitive Impairment and Amnestic Mild Cognitive Impairment Patients. Front Neurol 2018;9:342.
29. Lerch JP, Worsley K, Shaw WP, Greenstein DK, Lenroot RK, Giedd J, Evans AC. Mapping anatomical correlations across cerebral cortex (MACACC) using cortical thickness from MRI. Neuroimage 2006;31:993-1003.

30. Curtin F, Schulz P. Multiple correlations and Bonferroni's correction. Biol Psychiatry 1998;44:775-7.

31. Craig AD. How do you feel--now? The anterior insula and human awareness. Nat Rev Neurosci 2009;10:59-70.

32. Chang LJ, Yarkoni T, Khaw MW, Sanfey AG. Decoding the role of the insula in human cognition: functional parcellation and large-scale reverse inference. Cereb Cortex 2013;23:739-49.

33. Taylor KS, Seminowicz DA, Davis KD. Two systems of resting state connectivity between the insula and cingulate cortex. Hum Brain Mapp 2009;30:2731-45.

34. Vogt BA. Cingulate cortex in the three limbic subsystems. Handb Clin Neurol 2019;166:39-51.

35. Vogt BA. Midcingulate cortex: Structure, connections, homologies, functions and diseases. J Chem Neuroanat 2016;74:28-46.

36. Feng N, Gao M, Wu J, Yang G, Piao R, Liu P. Higher inter-hemispheric homotopic connectivity in lifelong premature ejaculation patients: a pilot resting-state fMRI study. Quant Imaging Med Surg 2021;11:3234-43.

37. Tan LL, Pelzer P, Heinl C, Tang W, Gangadharan V, Flor H, Sprengel R, Kuner T, Kuner R. A pathway from midcingulate cortex to posterior insula gates nociceptive hypersensitivity. Nat Neurosci 2017;20:1591-601.

38. Raichle ME. The brain's default mode network. Annu Rev Neurosci 2015;38:433-47.

39. Leech R, Kamourieh S, Beckmann CF, Sharp DJ. Fractionating the default mode network: distinct contributions of the ventral and dorsal posterior cingulate cortex to cognitive control. J Neurosci 2011;31:3217-24.

40. Leech R, Braga R, Sharp DJ. Echoes of the brain within the posterior cingulate cortex. J Neurosci 2012;32:215-22.

41. Wolff S, Kohrs C, Angenstein N, Brechmann A. Dorsal posterior cingulate cortex encodes the informational value of feedback in human-computer interaction. Sci Rep 2020;10:13030.

42. Seminowicz DA, Moayedi M. The Dorsolateral Prefrontal Cortex in Acute and Chronic Pain. J Pain 2017;18:1027-35.

43. Tillisch K, Mayer EA, Labus JS. Quantitative metaanalysis identifies brain regions activated during rectal distension in irritable bowel syndrome. Gastroenterology 
2011;140:91-100.

44. Lorenz J, Minoshima S, Casey KL. Keeping pain out of mind: the role of the dorsolateral prefrontal cortex in pain modulation. Brain 2003;126:1079-91.

45. Sestieri C, Corbetta M, Romani GL, Shulman GL. Episodic memory retrieval, parietal cortex, and the default mode network: functional and topographic analyses. J Neurosci 2011;31:4407-20.

46. Laird AR, Eickhoff SB, Li K, Robin DA, Glahn DC, Fox PT. Investigating the functional heterogeneity of the default mode network using coordinate-based metaanalytic modeling. J Neurosci 2009;29:14496-505.

47. Leung MK, Chan CC, Yin J, Lee CF, So KF, Lee TM. Increased gray matter volume in the right angular and posterior parahippocampal gyri in loving-kindness meditators. Soc Cogn Affect Neurosci 2013;8:34-9.

48. Wu H, Sun H, Wang C, Yu L, Li Y, Peng H, Lu X, Hu Q, Ning Y, Jiang T, Xu J, Wang J. Abnormalities in the structural covariance of emotion regulation networks in major depressive disorder. J Psychiatr Res 2017;84:237-42.

49. Buvanendran A, Ali A, Stoub TR, Kroin JS, Tuman KJ. Brain activity associated with chronic cancer pain. Pain Physician 2010;13:E337-42.

50. Koyama T, McHaffie JG, Laurienti PJ, Coghill RC. The subjective experience of pain: where expectations become reality. Proc Natl Acad Sci U S A 2005;102:12950-5.

51. York GK, Steinberg DA. Hughlings Jackson's theory of cerebral localization. J Hist Neurosci 1994;3:153-68.

52. Gale DJ, Flanagan JR, Gallivan JP. Human somatosensory cortex is modulated during motor planning. J Neurosci 2021;41:5909-22.

53. Predel C, Kaminski E, Hoff M, Carius D, Villringer A, Ragert P. Motor Skill Learning-Induced Functional Plasticity in the Primary Somatosensory Cortex: A Comparison Between Young and Older Adults. Front Aging Neurosci 2020;12:596438.

54. Hu L, Zhang L, Chen R, Yu H, Li H, Mouraux A. The primary somatosensory cortex and the insula contribute differently to the processing of transient and sustained nociceptive and non-nociceptive somatosensory inputs. Hum Brain Mapp 2015;36:4346-60.

55. Alexander GE, Crutcher MD. Functional architecture of basal ganglia circuits: neural substrates of parallel processing. Trends Neurosci 1990;13:266-71.

56. Pu R, Wu Z, Yu W, He H, Zhou Z, Wang Z, Zhong J. The association of myelination in the internal capsule with iron deposition in the basal ganglia in macaques: a magnetic resonance imaging study. Quant Imaging Med Surg 2020;10:1526-39.

57. Chudler EH, Dong WK. The role of the basal ganglia in nociception and pain. Pain 1995;60:3-38.

58. Emmert K, Breimhorst M, Bauermann T, Birklein F, Van De Ville D, Haller S. Comparison of anterior cingulate vs. insular cortex as targets for real-time fMRI regulation during pain stimulation. Front Behav Neurosci 2014;8:350.

59. Vassal M, Charroud C, Deverdun J, Le Bars E, Molino F, Bonnetblanc F, Boyer A, Dutta A, Herbet G, MoritzGasser S, Bonafé A, Duffau H, de Champfleur NM. Recovery of functional connectivity of the sensorimotor network after surgery for diffuse low-grade gliomas involving the supplementary motor area. J Neurosurg 2017;126:1181-90.

60. De Winter BY, Deiteren A, De Man JG. Novel nervous system mechanisms in visceral pain. Neurogastroenterol Motil 2016;28:309-15.

61. Menon V, Uddin LQ. Saliency, switching, attention and control: a network model of insula function. Brain Struct Funct 2010;214:655-67.

62. Etkin A, Wager TD. Functional neuroimaging of anxiety: a meta-analysis of emotional processing in PTSD, social anxiety disorder, and specific phobia. Am J Psychiatry 2007;164:1476-88.

63. Etkin A, Prater KE, Schatzberg AF, Menon V, Greicius MD. Disrupted amygdalar subregion functional connectivity and evidence of a compensatory network in generalized anxiety disorder. Arch Gen Psychiatry 2009;66:1361-72.

64. Stein MB, Simmons AN, Feinstein JS, Paulus MP. Increased amygdala and insula activation during emotion processing in anxiety-prone subjects. Am J Psychiatry 2007;164:318-27.

65. Baur V, Hänggi J, Langer N, Jäncke L. Resting-state functional and structural connectivity within an insulaamygdala route specifically index state and trait anxiety. Biol Psychiatry 2013;73:85-92.

66. Rolls ET. The cingulate cortex and limbic systems for emotion, action, and memory. Brain Struct Funct 2019;224:3001-18.

67. Caruana F, Gerbella M, Avanzini P, Gozzo F, Pelliccia V, Mai R, Abdollahi RO, Cardinale F, Sartori I, Lo Russo G, Rizzolatti G. Motor and emotional behaviours elicited by electrical stimulation of the human cingulate cortex. Brain 2018;141:3035-51.

68. Rolls ET. Limbic systems for emotion and for memory, 
but no single limbic system. Cortex 2015;62:119-57.

69. Dixon ML, Thiruchselvam R, Todd R, Christoff K.

Emotion and the prefrontal cortex: An integrative review. Psychol Bull 2017;143:1033-81.

Cite this article as: Zhang S, Chen F, Wu J, Liu C, Yang G, Piao R, Geng B, Xu K, Liu P. Altered structural covariance and functional connectivity of the insula in patients with Crohn's disease. Quant Imaging Med Surg 2022;12(2):1020-1036. doi: 10.21037/qims-21-509
70. Robinson OJ, Pike AC, Cornwell B, Grillon C. The translational neural circuitry of anxiety. J Neurol Neurosurg Psychiatry 2019;90:1353-60. 